\title{
Colecistectomía laparoscópica durante el embarazo, experiencia de un hospital universitario del noreste de México
}

\author{
Karla C. Guerra-Leza ${ }^{1}$, Marcela A. Narro-Martínez, \\ Aurora N. Ponce-Escobedo ${ }^{1}$ y José R. Fernández-Treviño ${ }^{1}$
}

\section{Laparoscopic cholecystectomy during pregnancy, experience from a University Hospital in northeast Mexico}

\begin{abstract}
Aim: To evaluate the safety of laparoscopic cholecystectomy during pregnancy, reporting complications and obstetric and perinatal outcomes in our institution. Materials and Method: Retrospective, observational study. Pregnant patients with a diagnosis of biliary pathology undergoing laparoscopic cholecystectomy were included. Sociodemographic, obstetric variables, complications or postoperative death were described. Results: 46 laparoscopic cholecystectomies were performed, mean age 25 years; $82.6 \%$ were in the second trimester of pregnancy; showing a complication rate of $4.4 \%$ directly related to the procedure with zero maternal mortality. Discussion: Biliary surgery is associated with a high rate of complications during pregnancy, however, there is more evidence supporting the idea of early intervention in symptomatic biliary disease, with a higher risk of fetal death under conservative management than under a laparoscopic approach, 14 as well as a higher recurrence ratecd. Surgery has been shown not to increase the risk of mortality for the mother and the fetus and its delay may result in an increase in morbidity in the short and long term. Conclusion: We observed a low rate of complications associated with the surgical procedure, as well as no maternal mortality.
\end{abstract}

Key words: cholecystitis; cholecystectomy; gallstones; pregnancy.

\section{Resumen}

Objetivo: Evaluar la seguridad de la colecistectomía laparoscópica durante el embarazo, reportando complicaciones y desenlaces obstétricos y perinatales en nuestra institución. Materiales y Método: Estudio retrospectivo, observacional. Se incluyeron pacientes embarazadas, con diagnóstico de patología biliar sometidas a colecistectomía laparoscópica. Se describieron variables sociodemográficas, obstétricas, complicaciones o muerte posoperatoria. Resultados: Se realizaron 46 colecistectomías laparoscópicas, edad media de 25 años; $82,6 \%$ cursaban el segundo trimestre de embarazo; mostrando tasa de complicaciones de 4,4\% directamente relacionadas con el procedimiento sin mortalidad materna. Discusión: Se asocia a la cirugía biliar a una tasa elevada de complicaciones durante el embarazo, sin embargo, existe mayor evidencia que apoya la idea de la intervención temprana en enfermedad biliar sintomática, con un mayor riesgo de muerte fetal bajo manejo conservador que bajo un abordaje quirúrgico, así como mayor tasa de recurrencia. Se ha demostrado que la cirugía no aumenta el riesgo de mortalidad para la madre y el feto y su retraso puede resultar en un incremento de morbilidad a corto y largo plazo. Conclusiones: Observamos una baja tasa de complicaciones asociadas al procedimiento quirúrgico, así como una nula mortalidad materna.

Palabras clave: colecistitis; colecistectomía; cálculos biliares; embarazo. 


\section{Introducción}

La colecistolitiasis es la enfermedad gastrointestinal que más frecuentemente requiere ingreso hospitalario $^{1}$. En México, el 20,5\% de los casos son en mujeres $^{2}$, hasta el $7 \%$ nulíparas y $20 \%$ multíparas ${ }^{3}$.

El embarazo es un estado prolitogénico, ya que el incremento del estrógeno eleva la producción de colesterol, mientras que la progesterona reduce la secreción de ácido biliar, inhibiendo el vaciamiento de la vesícula biliar 4 , con formación de lodo en $31 \%$ y de cálculos en $2 \%$ de los $\operatorname{casos}^{5}$. La colecistitis es una de las urgencias quirúrgicas más comunes en este grupo, con una incidencia del $0,05 \%$ al $0,8 \%{ }^{1}$. Actualmente, la colecistectomía es la segunda cirugía más común en mujeres embarazadas y se requiere en $45 \%$ de las pacientes 6 .

Registros históricos muestran que la cirugía biliar durante el embarazo se asocia a una tasa elevada de complicaciones maternofetales, por lo que se ha manejado tradicionalmente de forma conservadora reservando el manejo quirúrgico para casos severos o en caso de falla del manejo conservador (27\%$36 \%)^{4,5}$.Sin embargo, este abordaje aumenta el riesgo de reincidencia y de reingreso hospitalario ${ }^{5}$, en donde cada episodio de cólico biliar implica la eventual aparición de complicaciones, como colecistitis y pancreatitis (23\%-39\%), con una mortalidad materno-fetal de hasta $50 \%{ }^{5}$. Algunos estudios sugieren realizar colecistectomía en pacientes embarazadas sintomáticas, ya que se ha identificado recurrencia de los síntomas en $92 \%$ en el primer trimestre, $64 \%$ en el segundo y $44 \%$ en el tercero 7 .

Tabla 1. Predictores clínicos de riesgo de coledocolitiasis en pacientes con colelitiasis sintomática propuestos por la American Society of Gastrointestinal Endoscopy (ASGE)

\begin{tabular}{|c|c|c|}
\hline \multirow[t]{3}{*}{ Predictores "muy fuertes" } & \multicolumn{2}{|c|}{ Presencia de lito en vía biliar por ultrasonido } \\
\hline & \multicolumn{2}{|l|}{ Clínica de colangitis aguda } \\
\hline & \multicolumn{2}{|l|}{ Bilirrubina $>4 \mathrm{mg} / \mathrm{dL}$} \\
\hline \multirow[t]{2}{*}{ Predictores "fuertes" } & \multicolumn{2}{|c|}{ Dilatación de coledoco por ultrasonido $(>6 \mathrm{~mm})$} \\
\hline & \multicolumn{2}{|l|}{ Bilirrubina $1,8-4 \mathrm{mg} / \mathrm{dL}$} \\
\hline \multirow[t]{3}{*}{ Predictores "moderados" } & \multicolumn{2}{|l|}{ Pancreatitis biliar } \\
\hline & \multicolumn{2}{|l|}{ Edad mayor a 55 años } \\
\hline & \multicolumn{2}{|c|}{$\begin{array}{l}\text { Prueba bioquímica hepática anormal que no sea } \\
\text { bilirrubinas }\end{array}$} \\
\hline Riesgo alto & Riesgo intermedio & Riesgo bajo \\
\hline $\begin{array}{l}\text { Un predictor "muy fuerte" } \\
\text { y/o dos predictores "fuertes" }\end{array}$ & $\begin{array}{l}\text { Un predictor "fuerte" y/o un } \\
\text { predictor "moderado" }\end{array}$ & Sin predictores \\
\hline
\end{tabular}

La seguridad de la colecistectomía laparoscópica y abierta en pacientes gestantes ha sido tema de estudio previamente. Dhupar et al. ${ }^{3}$, compararon desenlaces asociados a la colecistectomía contra el manejo no quirúrgico en mujeres gestantes, encontrando menor tasa de complicaciones obstétricas (preeclampsia, ruptura prematura de membranas y parto pretérmino) en el grupo tratado quirúrgicamente $(18 \%)$ que el conservador $(36 \%)$.

De acuerdo con la literatura, la colecistectomía laparoscópica es igual de eficaz que la técnica abierta, sin embargo, aún surgen dudas acerca del costo-beneficio del tratamiento quirúrgico. Por ello, el objetivo de nuestro estudio es reportar nuestra experiencia en el manejo laparoscópico de la colecistolitiasis en el embarazo.

\section{Materiales y Método}

Se realizó un estudio retrospectivo y observacional de enero de 2016 a diciembre de 2018 en el Hospital Universitario "Dr. José Eleuterio González" en Monterrey, Nuevo León. Se estudiaron pacientes gestantes, con diagnóstico de patología biliar, sometidas a colecistectomía laparoscópica; definiendo la patología biliar no complicada como el cólico biliar sin datos de inflamación sistémica, signo de Murphy negativo, sin alteración en estudios de laboratorio, con datos de litiasis o lodo biliar por ultrasonido, sin engrosamiento de pared o dilatación del conducto colédoco; colecistitis aguda como dolor en hipocondrio derecho, signo de Murphy positivo, con datos sistémicos de inflamación o presencia de aumento de edema de la pared vesicular (>3 mm) y/o líquido perivesicular por imagenología.

Se incluyeron también pacientes con resolución de cuadro de pancreatitis biliar (identificado por dolor transfixiante en epigastrio, acompañado de náuseas o vómito, y alguno de los siguientes: aumento del valor de la amilasa sérica 3 o más veces el límite superior o datos sugerentes por imagen). El grado de obstrucción de vías biliares o riesgo de coledocolitiasis se determinó con base en los criterios de la American Society of Gastrointestinal Endoscopy (ASGE) (Tabla 1) ${ }^{8}$. Se excluyeron de este estudio pacientes con enfermedades hepáticas subyacentes.

Se recabaron las variables: edad, semanas de gestación, diagnóstico clínico, complicaciones y desenlaces obstétricos y perinatales. Se consideró como parto pretérmino a aquel producido entre 22 y 36 semanas con 6 días de gestación, así como aborto espontáneo a la pérdida de producto antes de la semana 20 de gestación o con un peso menor a los 
500 g. Para la valoración del tiempo de gestación, se consideró primer trimestre desde la concepción hasta las 13 semanas con 6 días, segundo trimestre de la semana 14 a la 27 con 6 días y tercer trimestre de la semana 28 en adelante.

Para la colecistectomía laparoscópica la técnica quirúrgica empleada fue la siguiente:

La paciente se coloca en decúbito lateral izquierdo a $30^{\circ}$ con ayuda de cuña, para evitar compresión de la vena cava por el útero grávido durante el procedimiento. Se indujo neumoperitoneo utilizando técnica de Hasson, hasta $12 \mathrm{mmHg}$. Se insertaron 4 trocares. T1 trocar de $10 \mathrm{~mm}$ a través de cicatriz umbilical, T2 trocar de $10 \mathrm{~mm}$ en epigastrio y 2 trocares de $5 \mathrm{~mm}$ subcostales derechos. En los casos en que el fondo uterino sobrepasaba la cicatriz umbilical se colocó primero el puerto epigástrico y luego el T1 1 o $2 \mathrm{~cm}$ por arriba de la cicatriz umbilical bajo visión directa.

En posición de Trendelenburg y rotación lateral izquierda se identificó y retrajo el fondo de vesícula hacia cefálico y bolsa de Hartmann hacia lateral izquierdo. Se disecó e identificó estructuras de triángulo hepato-cístico (borde hepático, conducto cístico y conducto colédoco) mediante visión crítica de seguridad. Se realizó ligadura de arteria y conducto cístico con clip metálico.

Se disecó vesícula de lecho hepático mediante técnica anterógrada y se extrajo pieza quirúrgica por puerto epigástrico. Se retiraron puertos mediante visión directa, el cierre de aponeurosis anterior de puerto epigástrico y umbilical fue realizado con vicryl 1-0, la piel fue cerrada con puntos absorbibles 3-0 o con nylon 3-0.

Este estudio fue aprobado por el comité de ética institucional, bajo el cumplimiento de regulaciones nacionales e internacionales.

Se realizó un análisis estadístico de las variables con el paquete estadístico SPSS versión 25 (Armonk, NY; IBM Corp.). Se evaluó la normalidad de la distribución de los datos de las variables cuantitativas con la prueba de Kolmogorov-Smirnov y se reportaron en media \pm desviación estándar o mediana (rango intercuartil), según corresponda.

\section{Resultados}

Durante el periodo de estudio, se realizaron un total de 46 colecistectomías laparoscópicas. La media de edad de las pacientes fue de 25,1 $\pm 6,4$ años, con una mínima de 13 años y una máxima de 41 , el $13 \%$ cursaba el primer trimestre de embarazo, $82,6 \%$ el segundo y 4,3\% el tercero (Tabla 2),
Tabla 2. Datos sociodemográficos de la población de estudio

\begin{tabular}{|lc|}
\hline Variable & \\
\hline Edad (años) & $25,1 \pm 6,4$ \\
Peso $(\mathrm{kg})$ & $67,7 \pm 14,7$ \\
Talla $(\mathrm{m})$ & $1,57 \pm 0,05$ \\
Índice de masa corporal & \\
Bajo peso & $1 \quad(2,2 \%)$ \\
Normopeso & $21(45,7 \%)$ \\
Sobrepeso & $12(26,1 \%)$ \\
Obesidad & $12(26,1 \%)$ \\
Comorbilidades & \\
Diabetes gestacional & $4(8,7 \%)$ \\
Cirugías previas & $2(4,3 \%)$ \\
Hipertensión arterial gestacional & $1(2,2 \%)$ \\
Otras & $5(10,9 \%)$ \\
Primigestas & $11(23,9 \%)$ \\
Evolución del embarazo actual & \\
Primer trimestre & $6(13 \%)$ \\
Segundo trimestre & $38(82,6 \%)$ \\
Tercer trimestre & $2(4,3 \%)$ \\
\hline
\end{tabular}

con un 23,9\% siendo primigestas al momento del procedimiento. $52 \%$ de las pacientes presentaban sobrepeso y/o algún grado de obesidad.

El $65,2 \%$ de las pacientes se ingresaron con el diagnóstico de patología biliar no complicada, $17,3 \%$ presentaron colecistitis aguda, 13\% pancreatitis biliar, 2,2\% coledocolitiasis resuelta por colangiopancreatografía retrógrada endoscópica (CPRE) y el 2,2\% con colecistolitiasis en la que se estableció, posteriormente, un diagnóstico de colestasis intrahepática del embarazo; con respecto a la sintomatología el 54\% de las pacientes habían presentado al menos un episodio previo de cólico biliar.

La mediana de tiempo quirúrgico fue de $55 \mathrm{mi}$ nutos. Un caso requirió conversión a procedimiento abierto y en dos fue necesario realizar colangiografía durante el procedimiento, por riesgo intermedio de coledocolitiasis. La tasa de complicaciones relacionadas con el procedimiento fue de $4,4 \%$, correspondiente a dos eventos: una lesión uterina grado I al momento de la colocación del trocar umbilical que fue resuelto transoperatoriamente sin mayores complicaciones y un caso con pérdida del producto durante las primeras 24 horas del posoperatorio. La media de tiempo de estancia hospitalaria fue de 8,1 $\pm 2,9$ días.

Se mantuvo el seguimiento de su embarazo en 
nuestra institución en $76,1 \%$ pacientes, con los siguientes desenlaces: $57,1 \%$ presentaron parto fisiológico, parto quirúrgico en 34,3\% y 8,6\% tuvieron pérdida del producto; lográndose un embarazo a término en el $80 \%$. La mortalidad materna fue nula en esta serie (Tabla 3).

\section{Discusión}

El diagnóstico y manejo de la litiasis vesicular en mujeres embarazadas ha sido un reto para los cirujanos; previamente, el embarazo fue considerado como contraindicación absoluta para la realización de cirugía laparoscópica, sin embargo, la literatura actual recomienda el manejo quirúrgico tanto para colecistitis aguda, como en pancreatitis aguda biliar (2 semanas posterior a la admisión), en mujeres no embarazadas 9 . El riesgo potencial de muerte fetal, la posibilidad de radiación fetal durante la CPRE, efectos mecánicos y fisiológicos de la colecistectomía laparoscópica, el riesgo anestésico y el efecto de los campos magnéticos en el feto durante la colangiopancreatografía por resonancia magnética dificultan la toma de decisiones en la paciente embarazada ${ }^{10}$. También existe evidencia de aumento del riesgo de peso bajo al nacer a consecuencia de prematurez y restricción de crecimiento intrauterina secundario a la cirugía en el embarazo ${ }^{11}$. Por ello, algunos cirujanos aún dudan sobre la realización de laparoscopía durante el embarazo, especialmente en el primer y tercer trimestre ${ }^{4}$.

Existe mayor evidencia que apoya la idea de la intervención temprana en enfermedad biliar sintomática en embarazadas, ya que hay un mayor riesgo de muerte fetal bajo manejo conservador, así como mayor tasa de recurrencia ${ }^{12}$.

En un periodo de tres años se realizaron 46 colecistectomías laparoscópicas en mujeres gestantes, la mayoría en el segundo trimestre y multigestas al momento del procedimiento. La indicación quirúrgica más común es la colecistitis aguda, seguida de colecistitis crónica, colelitiasis sintomática, pancreatitis biliar y cólico biliar recurrente ${ }^{4}$. En nuestro centro, las indicaciones más frecuentes fueron cólico biliar y colecistitis aguda, abarcando el 82,5\% de las indicaciones para este procedimiento. La tasa de complicaciones asociadas al procedimiento fue del $4,4 \%$, incluyendo un caso de pérdida fetal $(2,2 \%)$. De acuerdo con una revisión de Nasioudis y cols. ${ }^{4}$, la tasa de complicaciones maternas intraoperatorias y posoperatorias se observan en $3,8 \%$ y $4 \%$, respectivamente.

En una revisión realizada por Sedaghat y cols. ${ }^{14}$, se encontró que la tasa de conversión a técnica convencional se observa hasta en un $3,8 \%{ }^{14}$, en nuestra serie de casos fue del 2,2\%. En este caso, la conversión se realizó en una paciente con pancreatitis biliar, en la cual no se logró una identificación óptima de la vía biliar mediante técnica laparoscópica.

Es importante destacar la nula mortalidad materna en nuestras pacientes. Un reciente metaanálisis de Anthwal y cols. ${ }^{15}$, demostró que la cirugía biliar en mujeres embarazadas no aumenta el riesgo de mortalidad para la madre y el feto, de 1,5\% vs. $1,4 \%$ bajo manejo conservador. Se reporta que el retraso de la colecistectomía en esta población puede resultar en un incremento de morbilidad a corto y largo plazo, con recurrencia de los síntomas en un $92 \%$ cuando el cuadro debuta en el $1^{\text {er }}$ trimestre, $64 \%$ en el segundo y $44 \%$ en el tercero. También se ha encontrado un $23 \%-39 \%$ de complicaciones pancreatobiliares en pacientes con tratamiento conservador ${ }^{1}$. En nuestra serie, 54\% había presentado síntomas previamente, por lo que también pudo haber sido considerado al momento de la elección del abordaje quirúrgico. Además de la baja tasa de complicaciones, se sugiere que la intervención quirúrgica en enfermedad biliar sintomática no emergente durante el embarazo puede ser beneficioso y disminuir morbilidad ${ }^{16}$.

Algunas de las debilidades de nuestro estudio fueron la pérdida de seguimiento de algunas pacientes intervenidas por laparoscopía durante su embarazo y la naturaleza retrospectiva de nuestro trabajo. También hay que considerar que la gran parte de las pacientes incluidas cursaban el segundo trimestre, en donde más se ha estudiado esta intervención. Es necesario crear un modelo de estudio multicéntrico, aumentando así el número de casos y poder detectar algunos factores de mal pronóstico asociados a la intervención quirúrgica en el primer y tercer trimestre, así como evaluar el verdadero beneficio del manejo quirúrgico en estas etapas del embarazo.

\section{Conclusión}

En el presente estudio describimos la experiencia del manejo laparoscópico de mujeres gestantes con patología biliar en los últimos 3 años, donde reportamos una baja tasa de complicaciones asociadas al procedimiento quirúrgico. La evidencia sugiere que la mujer embarazada puede beneficiarse de esta intervención, sin embargo, hace falta mayor número de estudios prospectivos que puedan identificar el grupo de pacientes que se asocien menos a desenlaces obstétricos y perinatales negativos. 


\section{Responsabilidades éticas}

Protección de personas y animales. Los autores declaran que para esta investigación no se han realizado experimentos en seres humanos ni en animales.
Confidencialidad de los datos. Los autores declaran que en este artículo no aparecen datos de pacientes.

Conflictos de interés: no hay.

\section{Bibliografía}

1. European Association for the Study of the Liver (EASL). Clinical Practice Guidelines on the prevention, diagnosis and treatment of gallstones. J Hepatol. 2016;65:146-81. DOI: $10.1016 / \mathrm{j}$. jhep.2016.03.005.

2. Albarrán Cázares J, Mendoza Márquez J, Nevarez Borunda H. Factores de riesgo para colecistolitiasis en pacientes jóvenes con un índice de masa corporal menor a 30. Cirujano General 2012;34:121-4.

3. Dhupar R, Smaldone GM, Hamad GG. Is There a Benefit to Delaying Cholecystectomy for Symptomatic Gallbladder Disease During Pregnancy? Surg Endosc. 2010;24:108-12. DOI: $10.1007 / \mathrm{s} 00464-009-0544-x$.

4. Nasioudis D, Tsilimigras D, Economopoulos KP. Laparoscopic cholecystectomy during pregnancy: A systematic review of 590 patients. Int $\mathrm{J}$ Surg. 2016;27:165-75. DOI: 10.1016/j. ijsu.2016.01.070.

5. Hedström J, Nilsson J, Andersson R, Andersson B. Changing management of gallstone-related disease in pregnancy-a retrospective cohort analysis. Scand J Gastroenterol. 2017;52:1016-21. DOI: $10.1080 / 00365521.2017 .1333627$.

6. Corneille MG, Gallup TM, Bening T, Wolf
SE, Brougher C, Myers JG, et al. The use of laparoscopic surgery in pregnancy: evaluation of safety and efficacy. Am J Surg. 2010;200:363-7. DOI: 10.1016/j. amjsurg.2009.09.022.

7. Pearl JP, Price RR, Tonkin AE, Richardson WS, Stefanidis D. SAGES Guidelines for the Use of Laparoscopy During Pregnancy. Surg Endosc. 2017;31:376782. DOI: $10.1007 / \mathrm{s} 00464-017-5637-3$.

8. ASGE Standards of Practice Committee, Maple JT, Ben-Menachem T, Anderson MA, Appalaneni V, Banerjee S, et al. The role of endoscopy in the evaluation of suspected choledocholithiasis. Gastrointest Endosc. 2010;71:1-9. Available from: DOI: 10.1016/j. gie.2009.09.041.

9. Jackson H, Granger S, Price R, Rollins M, Earle D, Richardson W, et al. Diagnosis and Laparoscopic Treatment of Surgical Diseases During Pregnancy: An Evidence-Based Review. Surg Endosc. 2008;22:1917-27. DOI: 10.1007/s00464008-9989-6.

10. Date RS, Kaushal M, Ramesh A. A review of the management of gallstone disease and its complications in pregnancy. Am J Surg. 2008;196:599-608. DOI: 10.1016/j. amjsurg.2008.01.015.

11. Mazze RI, Källén B. Reproductive outcome after anesthesia and operation during pregnancy: A registry study of 5405 cases. Am J Obstet Gynecol. 1989;161:1178-85. DOI: 10.1016/00029378(89)90659-5.

12. Jelin EB, Smink DS, Vernon AH, Brooks DC. Management of biliary tract disease during pregnancy: A decision analysis. Surg Endosc. 2008;22:54-60.

13. Othman MO, Stone E, Hashimi M, Parasher G. Conservative management of cholelithiasis and its complications in pregnancy is associated with recurrent symptoms and more emergency department visits. Gastrointest Endosc. 2012;76:564-9. DOI: 10.1016/j. gie.2012.04.475.

14. Sedaghat N, Cao AM, Eslick GD, Cox MR. Laparoscopic Versus Open Cholecystectomy in Pregnancy: A Systematic Review and MetaAnalysis. Surg Endosc. 2017;31:673-9. DOI: 10.1007/s00464-016-5019-2.

15. Athwal R, Bhogal RH, Hodson J, Ramcharan S. Surgery for gallstone disease during pregnancy does not increase fetal or maternal mortality: a meta-analysis. Hepatobiliary Surg Nutr. 2016;5:53-7. DOI: 10.3978/j.issn.23043881.2015.11.02.

16. Yarmuch J. Cirugía y embarazo. Rev Chil Cir. 2010;62:520-4. doi.org/10.4067/ S0718-40262010000500018. 